\title{
Finite volume QCD at fixed topological charge
}

\author{
Sinya Aoki, ${ }^{1,2}$ Hidenori Fukaya, ${ }^{3}$ Shoji Hashimoto, ${ }^{4,5}$ and Tetsuya Onogi ${ }^{6}$ \\ ${ }^{1}$ Graduate School of Pure and Applied Sciences, University of Tsukuba, Tsukuba 305-8571, Ibaraki Japan \\ ${ }^{2}$ Riken BNL Research Center, Brookhaven National Laboratory, Upton, New York 11973, USA \\ ${ }^{3}$ Theoretical Physics Laboratory, RIKEN, Wako 351-0198, Japan \\ ${ }^{4}$ High Energy Accelerator Research Organization (KEK), Tsukuba 305-0801, Japan \\ ${ }^{5}$ School of High Energy Accelerator Science, The Graduate University for Advanced Studies (Sokendai), Tsukuba 305-0801, Japan \\ ${ }^{6} Y u k a w a$ Institute for Theoretical Physics, Kyoto University, Kyoto 606-8502, Japan
}

(Received 5 July 2007; published 19 September 2007)

\begin{abstract}
In finite volume the partition function of QCD with a given $\theta$ is a sum of different topological sectors with a weight primarily determined by the topological susceptibility. If a physical observable is evaluated only in a fixed topological sector, the result deviates from the true expectation value by an amount proportional to the inverse space-time volume $1 / V$. Using the saddle point expansion, we derive formulas to express the correction due to the fixed topological charge in terms of a $1 / V$ expansion. Applying this formula, we propose a class of methods to determine the topological susceptibility in QCD from various correlation functions calculated in a fixed topological sector.
\end{abstract}

DOI: 10.1103/PhysRevD.76.054508

PACS numbers: 12.38.Gc, 11.15.Ha

\section{INTRODUCTION}

Quantum chromodynamics (QCD) in four space-time dimensions allows topologically nontrivial gauge configurations labeled by a winding number or a topological charge $Q$. The path integral to define the partition function of QCD includes an integral over configurations with arbitrary $Q$. In order to ensure the cluster decomposition property of physical observables, the weight among different topological sectors must be $e^{i \theta Q}$, which defines the $\theta$ vacuum of QCD. If one considers a path integral restricted in a fixed topological sector, the cluster decomposition property-one of the necessary properties of quantum field theory - is violated [1]. In this paper we address the question of fixing topology in the context of nonperturbative calculation of QCD on the lattice. But the analysis does not depend on any particular regularization of the theory.

In the lattice QCD simulations the inclusion of the effects of dynamical quarks is computationally most demanding. Since the direct computation of the fermion determinant $\operatorname{det}(D+m)^{2}$, with $D$ the Dirac operator on the lattice and $m$ the quark mass, requires prohibitive computational cost, one usually introduces a pseudofermion field $\phi$ to write the determinant in the form $\int[d \phi] \times$ $\left[d \phi^{\dagger}\right] \exp \left(-\phi^{\dagger}(D+m)^{-2} \phi\right)$, so that the problem is reduced to an evaluation of the inverse fermion matrix $(D+$ $m)^{-1}$. Since the effective lattice action becomes nonlocal, the Monte Carlo updation is most efficiently done by updating all the gauge links on the lattice at the same time introducing a molecular dynamics evolution. The most popular such algorithm to date is the hybrid Monte Carlo algorithm [2] that combines the molecular dynamics evolution with a Metropolis accept/reject step.

With the molecular dynamics evolution, the ergodicity becomes a potential problem when there exist more than one region of phase space that are separated by some potential wall, because the "kinetic energy" of the molecular dynamics system may not be enough to go through the potential wall. This situation happens for QCD in four dimensions because of the nontrivial topological sectors. In the continuum theory the potential barrier is infinite and the gauge configurations in different topological sectors cannot be reached by a single stream of the continuous evolution. On the lattice, the potential barrier is finite (order of inverse lattice spacing $1 / a$ ) and the probability of tunneling among different topological sectors is nonzero, but will exponentially drop ( $\sim e^{-U_{0} / a}$ with $U_{0} / a$ a nominal potential height) as the continuum limit is approached. This means that the correct sampling of topological charge and thus the valid simulation of the $\theta$ vacuum of QCD will become increasingly more difficult $[3,4]$. In fact, this problem already manifests itself in the recent dynamical overlap fermion simulations by the JLQCD Collaboration [5-8], since they explicitly introduce a term that prevents topology change [9] in order to avoid large numerical cost due to the discontinuity of the overlap operator along the topology boundary.

One may then ask whether lattice QCD simulations with a fixed topological charge are useful, in general, to extract physics of real world, i.e. QCD at a given value of $\theta$. (If the $C P$ symmetry is preserved, $\theta=0$. Small but nonzero $\theta$ implies an interesting physics case to give rise to the neutron electric dipole moment.) The fixed topology simulation is desirable in the study of the $\epsilon$ regime of QCD, since the physical quantities have striking dependence on the topological charge, which is an important part of the physics we are interested in. (For a recent unquenched lattice simulation in the $\epsilon$ regime, see $[10,11]$.) But in the $p$ regime, the fixed topology simulation gives rise to a systematic effect. In this paper we try to answer this question theoretically without relying on any particular model 
or assumption. Extending a previous work by Brower et al. [12], we show that observables calculated at a fixed topological charge are different from those at $\theta=0$ by an amount of order of inverse space-time volume $1 / V$, so that both agree in the infinite volume limit.

This result can be understood intuitively within the instanton picture of the topological excitation in the QCD vacuum. The instanton is a local object carrying a unit topological charge. Starting from a trivial topological sector, an instanton and anti-instanton can be pair-created or annihilated without changing the net topology and then may be separated from each other. If we look at a region (or subvolume) including the (anti-)instanton, that region has locally nontrivial topological charge. In this way, the topological fluctuation may occur even if the net topological charge is kept fixed. Supporting evidence of this expectation has been found in the chirality density of low-lying eigenmodes of the overlap-Dirac operator; i.e. local chirality is strongly peaked at \pm 1 even on the gauge configurations with net topological charge $Q=0$ [6]. The effect of fixing the net topology will become relatively small as the total volume becomes larger, as there is more chance to create (or annihilate) the instanton-anti-instanton pairs. The topological susceptibility $\chi_{t}$ characterizes the magnitude of these local topological fluctuations.

In fact, in our formulation the topological susceptibility enters in the difference of $O(1 / V)$ between the fixed topological sector and the fixed $\theta$ vacuum. This type of finite volume effect can even be evaluated, once we know the coefficient of $1 / V$, which is found, in general, to be a second derivative of the physical quantity of interest with respect to $\theta$, as well as the topological susceptibility $\chi_{t}$. In this paper, we describe the method to extract $\chi_{t}$ from a gauge ensemble in the fixed topological sector, leaving the estimate of the finite size effects for other physical quantities for a future publication.

This paper is organized as follows. In Sec. II, by using the saddle point expansion, we derive a formula which expresses the partition function at a fixed topological charge in terms of the partition function at $\theta=0$ and its derivatives with respect to $\theta$. We extend this formula to the case for arbitrary correlation functions. From these results it is easy to show that arbitrary correlation functions at fixed topological charge agree with those at $\theta=0$ in the infinite volume limit. In Sec. III, as an application of the above formulas, we show that the topological susceptibility $\chi_{t}$ can be extracted from the two-point function of the topological charge density at a fixed topological charge. Through the Ward-Takahashi (WT) identities, we then relate the two-point function of the topological charge density with a two-point function of the pseudoscalar density, which is more suitable for actual calculations with the overlap fermions. An extension to the case of four- and three-point correlation functions is also discussed. In Sec. IV, we consider a case of $C P$-odd observ- ables, taking the calculation of the neutron electric dipole moment as an example. Our conclusion is given in Sec. V. In Appendix A we check the validity of the saddle point expansion, by comparing the expansion with an exact calculation for a simple example. Results at order $V^{-3}$ are summarized in Appendix B.

\section{GENERAL FORMULA}

\section{A. Partition function at a fixed topological charge}

We first consider the partition function in the $\theta$ vacuum defined by

$$
Z(\theta) \equiv\langle\theta \mid \theta\rangle=\exp [-V E(\theta)]
$$

where $E(\theta)$ is the energy per (space-time) volume $V$. Without loss of generality we take $Z(0)=1$ as a normalization, which is equivalent to $E(0)=0 . E(\theta)$ satisfies the conditions $E(\theta+2 \pi)=E(\theta)=E(-\theta)$. The topological susceptibility $\chi_{t}$ at $\theta=0$ is defined by

$$
\chi_{t}=\frac{\left\langle 0\left|Q^{2}\right| 0\right\rangle}{V},
$$

which is obtained by a second derivative of $E(\theta)$,

$$
\chi_{t}=\left.\frac{d^{2} E(\theta)}{d \theta^{2}}\right|_{\theta=0} .
$$

Since $\chi_{t} \geq 0$ by definition, $\theta=0$ is a local minimum of $E(\theta)$. Moreover, Vafa and Witten proved that $Z(0)>Z(\theta)$ [13], which leads to $E(0)<E(\theta)$ for $\forall \theta \neq 0$. Namely, $\theta=0$ is the global minimum of the function $E(\theta)$. While we assume that $E(\theta)$ is analytic at $\theta=0, E(\theta)$ may have a nonanalyticity at $\theta \neq 0$, in particular, at $\theta=\pi$. For instance, chiral perturbation theory (ChPT) at the leading order gives

$$
E(\theta)=\chi_{t} N_{f}^{2}\left[1-\cos \left(\theta / N_{f}\right)\right], \quad-\pi<\theta \leq \pi
$$

for $N_{f}$ number of flavors. A $\theta$ derivative of this particular form is discontinuous at $\theta= \pm \pi$ for $N_{f}>1$ [14]. From these general conditions we can expand $E(\theta)$ around $\theta=$ 0 ,

$$
E(\theta)=\sum_{n=1}^{\infty} \frac{c_{2 n}}{(2 n) !} \theta^{2 n}=\frac{\chi_{t}}{2} \theta^{2}+O\left(\theta^{4}\right) .
$$

The partition function at a fixed topological charge $Q$ is a Fourier coefficient of the periodic function $Z(\theta)$,

$$
\begin{aligned}
Z_{Q} & =\frac{1}{2 \pi} \int_{-\pi}^{\pi} d \theta Z(\theta) \exp (i \theta Q) \\
& =\frac{1}{2 \pi} \int_{-\pi}^{\pi} d \theta \exp (-V F(\theta)),
\end{aligned}
$$

where $F(\theta) \equiv E(\theta)-i \theta Q / V$, and the Fourier expansion of $Z(\theta)$ is written as 


$$
Z(\theta)=\sum_{Q=0, \pm 1, \pm 2, \cdots} Z_{Q} e^{-i \theta Q} .
$$

For a large enough volume, we can evaluate the $\theta$ integral in (6) by the saddle point expansion. The saddle point $\theta_{c}$ is given by

$$
\theta_{c}=i \frac{Q}{\chi_{t} V}\left(1+O\left(\delta^{2}\right)\right)
$$

where $\delta \equiv Q /\left(\chi_{t} V\right)$. We then expand $F(\theta)$ as

$$
\begin{aligned}
F(\theta)= & F\left(\theta_{c}\right)+\frac{E^{(2)}}{2}\left(\theta_{c}\right)\left(\theta-\theta_{c}\right)^{2} \\
& +\sum_{n=3}^{\infty} \frac{E^{(n)}\left(\theta_{c}\right)}{n !}\left(\theta-\theta_{c}\right)^{n},
\end{aligned}
$$

where $E^{(n)}$ is the $n$th derivative of $E(\theta)$ with respect to $\theta$ at $\theta=\theta_{c}$, and is given by

$$
\begin{gathered}
V F\left(\theta_{c}\right)=\frac{Q^{2}}{2 \chi_{t} V}\left(1+O\left(\delta^{2}\right)\right), \\
E^{(2)}\left(\theta_{c}\right)=\chi_{t}\left(1+O\left(\delta^{2}\right)\right), \\
E^{(2 n)}\left(\theta_{c}\right)=c_{2 n}\left(1+O\left(\delta^{2}\right)\right), \\
E^{(2 n-1)}\left(\theta_{c}\right)=\theta_{c} c_{2 n}\left(1+O\left(\delta^{2}\right)\right) .
\end{gathered}
$$

Hereafter we omit an argument $\theta_{c}$ of $E^{(n)}\left(\theta_{c}\right)$ and simply write it as $E^{(n)}$ unless otherwise stated. By a change of variable $s=\sqrt{E^{(2)} V}\left(\theta-\theta_{c}\right)$ we can rewrite the integral as

$$
\begin{aligned}
Z_{Q} & =\frac{e^{-V F\left(\theta_{c}\right)}}{2 \pi \sqrt{E^{(2)} V}} \int_{\sqrt{E^{(2)} V\left(-\pi-\theta_{c}\right)}}^{\sqrt{E^{(2)} V}\left(\pi-\theta_{c}\right)} d s \exp \left[-\frac{s^{2}}{2}-\sum_{n=3} \frac{E^{(n)} V}{n !}\left(\frac{s}{\sqrt{E^{(2)} V}}\right)^{n}\right] \\
& =\frac{e^{-V F\left(\theta_{c}\right)}}{2 \pi \sqrt{E^{(2)} V}} \int_{-\infty}^{\infty} d s \exp \left[-\frac{s^{2}}{2}-\sum_{n=3} \frac{E^{(n)} V}{n !}\left(\frac{s}{\sqrt{E^{(2)} V}}\right)^{n}\right]+O\left(e^{-V}\right) \\
& =\frac{e^{-V F\left(\theta_{c}\right)}}{\sqrt{2 \pi E^{(2)} V}}\left\langle\exp \left[-\sum_{n=3} \frac{E^{(n)} V}{n !}\left(\frac{s}{\sqrt{E^{(2)} V}}\right)^{n}\right]\right\rangle+O\left(e^{-V}\right) .
\end{aligned}
$$

We defined

$$
\langle f(s)\rangle=\frac{1}{\sqrt{2 \pi}} \int_{-\infty}^{\infty} d s e^{-s^{2} / 2} f(s),
$$

with which $\left\langle s^{2 n}\right\rangle=(2 n-1)$ !!. Neglecting exponentially suppressed terms and expanding in powers of $1 / V$, we obtain

$$
\begin{aligned}
Z_{Q} & =\frac{e^{-V F\left(\theta_{c}\right)}}{\sqrt{2 \pi E^{(2)} V}}\left[1-\left\langle\sum_{n=3} \frac{E^{(n)} V}{n !}\left(\frac{s}{\sqrt{E^{(2)} V}}\right)^{n}\right\rangle+\cdots\right] \\
& =\frac{e^{-V F\left(\theta_{c}\right)}}{\sqrt{2 \pi E^{(2)} V}}\left[1-\frac{E^{(4)} V}{8\left(E^{(2)} V\right)^{2}}+O\left(\frac{1}{V^{2}}\right)\right] \\
& =\frac{1}{\sqrt{2 \pi \chi_{t} V}} \exp \left[-\frac{Q^{2}}{2 \chi_{t} V}\right]\left[1-\frac{c_{4}}{8 V \chi_{t}^{2}}+O\left(\frac{1}{V^{2}}, \delta^{2}\right)\right] .
\end{aligned}
$$

This shows that, as long as $\delta \ll 1$ (equivalently, $Q \ll$ $\chi_{t} V$ ), the distribution of $Q$ becomes the Gaussian distribution. Note, however, that the distribution can deviate from a Gaussian for $Q=O(V)$, as numerically observed on a quenched lattice [15-18].

\section{B. Correlation functions}

Here we consider an arbitrary correlation function

$$
G(\theta)=\left\langle\theta\left|O_{1} O_{2} \cdots O_{n}\right| \theta\right\rangle,
$$

whose Fourier coefficient at a fixed topological charge $Q$ is defined by

$$
G_{Q}=\frac{1}{Z_{Q}} \frac{1}{2 \pi} \int d \theta Z(\theta) G(\theta) \exp (i \theta Q) .
$$

Note that the operators $O_{i}$ do not contain $\theta$, and the $\theta$ dependence comes solely from the vacuum angle. Using the saddle point expansion as before, we obtain

$$
\begin{aligned}
G_{Q}= & \frac{1}{Z_{Q}} \frac{e^{-V F\left(\theta_{c}\right)}}{2 \pi \sqrt{E^{(2)} V}} \int_{-\infty}^{\infty} d s \exp \left[-\frac{s^{2}}{2}-\sum_{n=3}^{\infty} \frac{E^{(n)} V}{n !}\right. \\
& \left.\times\left(\frac{s}{\sqrt{E^{(2)} V}}\right)^{n}\right] G\left(\theta_{c}+\frac{s}{\sqrt{E^{(2)} V}}\right) \\
= & G\left(\theta_{c}\right)+\sum_{k=1}^{\infty} G^{(k)}\left(\theta_{c}\right) \frac{1}{k !}\left\langle\left\langle\left(\frac{s}{\sqrt{E^{(2)} V}}\right)^{k}\right\rangle\right\rangle,
\end{aligned}
$$

where we define

$$
\begin{aligned}
\langle\langle f(s)\rangle\rangle \equiv & \frac{1}{Z_{Q}} \frac{e^{-V F\left(\theta_{c}\right)}}{2 \pi \sqrt{E^{(2)} V}} \int_{-\infty}^{\infty} d s f(s) \\
& \times \exp \left[-\frac{s^{2}}{2}-\sum_{n=3}^{\infty} \frac{E^{(n)} V}{n !}\left(\frac{s}{\sqrt{E^{(2)} V}}\right)^{n}\right]
\end{aligned}
$$

and $G^{(n)}$ is the $n$th derivative of $G$ with respect to $\theta$. Using the formulas

$$
\langle\langle 1\rangle\rangle=1,
$$




$$
\begin{gathered}
\left\langle\left\langle s^{2}\right\rangle\right\rangle=1-\frac{E^{(4)} V}{2\left(E^{(2)} V\right)^{2}}+\frac{5\left(E^{(3)} V\right)^{2}}{4\left(E^{(2)} V\right)^{3}}+O\left(V^{-2}\right), \\
\left\langle\left\langle s^{4}\right\rangle\right\rangle=3 ! !+O\left(V^{-1}\right), \\
\langle\langle s\rangle\rangle=-\frac{E^{(3)} V}{2\left(E^{(2)} V\right)^{3 / 2}}\left(1-\frac{4 E^{(4)} V}{3\left(E^{(2)} V\right)^{2}}+\frac{5\left(E^{(3)} V\right)^{2}}{4\left(E^{(2)} V\right)^{3}}\right)-\frac{E^{(5)} V}{8\left(E^{(2)} V\right)^{5 / 2}}+O\left(V^{-5 / 2}\right), \\
\left\langle\left\langle s^{3}\right\rangle\right\rangle=-\frac{5 E^{(3)} V}{2\left(E^{(2)} V\right)^{3 / 2}}+O\left(V^{-3 / 2}\right),
\end{gathered}
$$

we finally obtain

$$
\begin{aligned}
G_{Q}= & G\left(\theta_{c}\right)+G^{(2)}\left(\theta_{c}\right) \frac{1}{2 E^{(2)} V}\left(1-\frac{E^{(4)} V}{2\left(E^{(2)} V\right)^{2}}+\frac{5\left(E^{(3)} V\right)^{2}}{4\left(E^{(2)} V\right)^{3}}\right)+G^{(4)}\left(\theta_{c}\right) \frac{1}{8\left(E^{(2)} V\right)^{2}} \\
& -G^{(1)}\left(\theta_{c}\right)\left[\frac{E^{(3)} V}{2\left(E^{(2)} V\right)^{2}}\left(1-\frac{4 E^{(4)} V}{3\left(E^{(2)} V\right)^{2}}+\frac{5\left(E^{(3)} V\right)^{2}}{4\left(E^{(2)} V\right)^{3}}\right)+\frac{E^{(5)} V}{8\left(E^{(2)} V\right)^{3}}\right]-G^{(3)}\left(\theta_{c}\right) \frac{5 E^{(3)} V}{12\left(E^{(2)} V\right)^{3}}+O\left(V^{-3}\right)
\end{aligned}
$$

The above expansion is valid for any $\theta_{c}$ as long as $G^{(n)}\left(\theta_{c}\right) / G\left(\theta_{c}\right)=O(1)$ as $V \rightarrow \infty$.

Depending on the size of $\theta_{c}$, we can further expand the above formula. If we take $\theta_{c}=O\left(V^{-1}\right)$ [equivalently, $Q=$ $O(1)]$, we have

$$
\begin{aligned}
G_{Q}= & G(0)+G^{(1)}(0) \theta_{c}+G^{(2)}(0) \frac{\theta_{c}^{2}}{2}+G^{(2)}(0) \frac{1}{2 E^{(2)} V}\left(1-\frac{E^{(4)} V}{2\left(E^{(2)} V\right)^{2}}\right)+G^{(3)} \frac{\theta_{c}}{2 E^{(2)} V}+G^{(4)}(0) \frac{1}{8\left(E^{(2)} V\right)^{2}} \\
& -G^{(1)}(0)\left[\frac{E^{(3)} V}{2\left(E^{(2)} V\right)^{2}}\left(1-\frac{4 E^{(4)} V}{3\left(E^{(2)} V\right)^{2}}\right)+\frac{E^{(5)} V}{8\left(E^{(2)} V\right)^{3}}\right]-G^{(2)}(0) \theta_{c} \frac{E^{(3)} V}{2\left(E^{(2)} V\right)^{2}}+O\left(V^{-3}\right) \\
= & G(0)+G^{(2)}(0) \frac{1}{2 \chi_{t} V}\left[1-\frac{Q^{2}}{\chi_{t} V}-\frac{c_{4}}{2 \chi_{t}^{2} V}\right]+G^{(4)}(0) \frac{1}{8 \chi_{t}^{2} V^{2}}+G^{(1)}(0) \frac{i Q}{\chi_{t} V}\left(1-\frac{c_{4}}{2 \chi_{t}^{2} V}\right)+G^{(3)}(0) \frac{i Q}{2 \chi_{t}^{2} V^{2}}+O\left(V^{-3}\right) .
\end{aligned}
$$

If $G$ is $C P$ even, $G$ is an even function of $\theta$, so that

$$
\begin{aligned}
G_{Q}^{\text {even }}= & G(0)+G^{(2)}(0) \frac{1}{2 \chi_{t} V}\left[1-\frac{Q^{2}}{\chi_{t} V}-\frac{c_{4}}{2 \chi_{t}^{2} V}\right] \\
& +G^{(4)}(0) \frac{1}{8 \chi_{t}^{2} V^{2}}+O\left(V^{-3}\right),
\end{aligned}
$$

while, if $G$ is $C P$ odd, we have

$$
\begin{aligned}
G_{Q}^{\mathrm{odd}}= & G^{(1)}(0) \frac{i Q}{\chi_{t} V}\left(1-\frac{c_{4}}{2 \chi_{t}^{2} V}\right)+G^{(3)}(0) \frac{i Q}{2 \chi_{t}^{2} V^{2}} \\
& +O\left(V^{-3}\right) .
\end{aligned}
$$

In order to claim that the above expansion is convergent, $G^{(n)}(0) / G(\theta)$ must be $O(1)$. This condition is satisfied because the expansion

$$
G(\theta)=G(0)+\sum_{n=1}^{\infty} G^{(n)}(0) \frac{\theta^{n}}{n !}
$$

is valid for $\forall \theta=O(1)$.
The formula (28) provides an estimate of the finite size effect due to the fixed topological charge. The leading correction is of order $O(1 / \mathrm{V})$ as advertised. The dimension is compensated by the topological susceptibility $\chi_{t}$. In ChPT it is evaluated as $\chi_{t}=m \Sigma / N_{f}$ by the sea quark mass $m$ and the chiral condensate $\Sigma$ as well as the number of flavors $N_{f}$ [19]. The finite volume correction is suppressed when the quark mass is larger than $1 /(\Sigma V)$, while it becomes significant when $m \sim 1 /(\Sigma V)$. This is consistent with the fact that the topological charge has a strong effect in the $\epsilon$ regime, which is characterized by $m \Sigma V \lesssim 1$. The correction has a coefficient $G^{(2)}(0)$ that represents the $\theta$ dependence of the correlator. This is not known, in general, but can be fitted with lattice data at various $Q$. In the mass region where ChPT is applicable, it can also be estimated, as done in [12] at the tree level for pseudoscalar meson mass. One-loop calculations are in progress.

The other interesting formula (29) suggests a possibility to calculate $C P$-odd observables, such as the neutron elec- 
tric dipole moment, at a fixed nonzero topological charge, which will be discussed in Sec. IV.

\section{TOPOLOGICAL SUSCEPTIBILITY}

In this section, we propose methods to extract the topological susceptibility $\chi_{t}$ from correlation functions at a fixed topological charge $Q$.

\section{A. Two-point correlation function}

\section{Bosonic formula}

Suppose that there is a well-defined local operator $\omega(x)$ that measures the local topological charge. The global topological charge $Q$ is then obtained as $Q=$ $\int d^{4} x \omega(x)$, and the topological susceptibility is $\chi_{t}=$ $\int d^{4} x\langle\omega(x) \omega(0)\rangle$, where the expectation value is taken for the $\theta=0$ vacuum.

Since $\omega(x) \omega(0)$ is $C P$ even, Eq. (28) gives

$$
\begin{aligned}
\langle\omega(x) \omega(0)\rangle_{Q}= & \langle\omega(x) \omega(0)\rangle+\langle\omega(x) \omega(0)\rangle^{(2)} \frac{1}{2 V \chi_{t}} \\
& \times\left(1-\frac{Q^{2}}{V \chi_{t}}-\frac{c_{4}}{2 \chi_{t}^{2} V}\right) \\
& +\langle\omega(x) \omega(0)\rangle^{(4)} \frac{1}{8 \chi_{t}^{2} V^{2}}+O\left(V^{-3}\right) .
\end{aligned}
$$

Here $\langle\mathcal{O}\rangle$ means a vacuum expectation value of $\mathcal{O}$ at $\theta=0$ and $\langle\mathcal{O}\rangle^{(n)}$ denotes its $n$th derivative with respect to $\theta$. In the large separation limit $|x| \rightarrow \infty$, the $C P$ invariance at $\theta=0$ and the clustering property at a fixed $\theta[1]$ gives

$$
\langle\omega(x) \omega(0)\rangle \rightarrow\langle\omega(x)\rangle\langle\omega(0)\rangle=0 .
$$

In addition,

$$
\langle\omega(x) \omega(0)\rangle^{(2)}=-\left\langle\omega(x) \omega(0) Q^{2}\right\rangle+\langle\omega(x) \omega(0)\rangle\left\langle Q^{2}\right\rangle,
$$

whose second term vanishes as $|x| \rightarrow \infty$. Denoting a connected vacuum expectation value (VEV) as $\langle\cdots\rangle_{c}$, the first term can be written as

$$
\begin{aligned}
\int d^{4} y d^{4} z\langle\omega(x) \omega(0) \omega(y) \omega(z)\rangle \\
=\int d^{4} y d^{4} z\left[\langle\omega(x) \omega(0) \omega(y) \omega(z)\rangle_{c}+\langle\omega(x) \omega(0)\rangle\right. \\
\quad \times\langle\omega(y) \omega(z)\rangle+\langle\omega(x) \omega(y)\rangle\langle\omega(0) \omega(z)\rangle \\
\quad+\langle\omega(x) \omega(z)\rangle\langle\omega(y) \omega(0)\rangle] .
\end{aligned}
$$

As $|x| \rightarrow \infty$, the first and the second terms vanish and we obtain

$$
\begin{gathered}
\int d^{4} y \int d^{4} z\langle\omega(x) \omega(y)\rangle\langle\omega(z) \omega(0)\rangle+(y \leftrightarrow z) \\
=2 \int d^{4} y\langle\omega(y) \omega(0)\rangle \int d^{4} z\langle\omega(x) \omega(z)\rangle .
\end{gathered}
$$

Using the translational invariance, this becomes $2 \chi_{t}^{2}$. Therefore, the term $\langle\omega(x) \omega(0)\rangle^{(2)}$ in (31) gives $-2 \chi_{t}^{2}$ in the large separation limit.

Similarly, we consider

$$
\begin{aligned}
\langle\omega(x) \omega(0)\rangle^{(4)}= & \left\langle\omega(x) \omega(0) Q^{4}\right\rangle-6\left\langle\omega(x) \omega(0) Q^{2}\right\rangle\left\langle Q^{2}\right\rangle \\
& +\langle\omega(x) \omega(0)\rangle\left\{6\left\langle Q^{2}\right\rangle^{2}-\left\langle Q^{4}\right\rangle\right\} .
\end{aligned}
$$

The first term is written as

$$
\begin{aligned}
\left\langle\omega(x) \omega(0) Q^{4}\right\rangle= & \int \prod_{i} d^{4} x_{i}\left\langle\omega(x) \omega(0) \omega\left(x_{1}\right) \omega\left(x_{2}\right) \omega\left(x_{3}\right)\right. \\
& \left.\times \omega\left(x_{4}\right)\right\rangle,
\end{aligned}
$$

which may be decomposed in terms of the connected VEVs. Since the terms containing both $\omega(x)$ and $\omega(0)$ in the same connected VEV vanish, only the following terms remain:

$$
\begin{aligned}
& \int \prod_{i} d^{4} x_{i}\left[8\left\langle\omega(x) \omega\left(x_{1}\right) \omega\left(x_{2}\right) \omega\left(x_{3}\right)\right\rangle_{c}\left\langle\omega(0) \omega\left(x_{4}\right)\right\rangle\right. \\
& \left.+12\left\langle\omega(x) \omega\left(x_{1}\right)\right\rangle\left\langle\omega\left(x_{2}\right) \omega\left(x_{3}\right)\right\rangle\left\langle\omega(0) \omega\left(x_{4}\right)\right\rangle\right] \\
& \quad=8 \chi_{t} \frac{\left\langle Q^{4}\right\rangle_{c}}{V}+12 V \chi_{t}^{3} .
\end{aligned}
$$

Putting this into (35) we have

$$
\langle\omega(x) \omega(0)\rangle^{(4)} \rightarrow 8 \chi_{t} \frac{\left\langle Q^{4}\right\rangle_{c}}{V}=\frac{8 \chi_{t}}{V} \frac{d^{4}}{d \theta^{4}} \log Z(\theta)=-8 \chi_{t} c_{4}
$$

for the fourth-derivative term in (31).

Gathering all terms we arrive at

$$
\begin{aligned}
\lim _{|x| \rightarrow \operatorname{large}}\langle\omega(x) \omega(0)\rangle_{Q}= & \frac{1}{V}\left(\frac{Q^{2}}{V}-\chi_{t}-\frac{c_{4}}{2 \chi_{t} V}\right)+O\left(V^{-3}\right) \\
& +O\left(e^{-m_{\eta^{\prime}}|x|}\right),
\end{aligned}
$$

where the flavor singlet pseudoscalar meson mass, $m_{\eta^{\prime}}$, is the lightest mass of possible intermediate states. This result explicitly shows that QCD at a fixed topological charge (in a finite volume) is sick as a quantum field theory, because the clustering property is violated. However, the magnitude of the violation can be estimated as a $1 / V$ expansion without introducing strong assumptions on the details of the dynamics of QCD. The same result (up to the $c_{4}$ term) was obtained in the context of a two-dimensional model in [20].

Physical quantities such as the topological susceptibility $\chi_{t}$ can be obtained through (39). In practice, this formula will be used for a finite separation $x$ instead of $|x| \rightarrow \infty$. The clustering property (32) in the $\theta$ vacuum receives a correction of order of $e^{-m_{\eta^{\prime}}|x|}$, which vanishes quickly because the flavor singlet meson $\eta^{\prime}$ acquires a large mass due to the axial anomaly of QCD. 


\section{Fermionic formula}

We now express the bosonic correlation function $\langle\omega(x) \omega(0)\rangle$ in terms of a fermionic one using the anomalous axial U(1) WT identities for an arbitrary operator $O$ :

$$
\left\langle\partial_{\mu} A_{\mu}(x) O-2 m P(x) O+2 \omega(x) O+\delta_{x} O\right\rangle=0,
$$

where $\quad A_{\mu}(x)=\frac{1}{N_{f}} \sum_{f} \bar{\psi}^{f}(x) \gamma_{\mu} \gamma_{5} \psi^{f}(x) \quad$ and $\quad P(x)=$ $\frac{1}{N_{f}} \sum_{f} \bar{\psi}^{f}(x) \gamma_{5} \psi^{f}(x)$ are the flavor singlet axial-vector current and pseudoscalar density, respectively, and $\delta_{x} O$ denotes an axial rotation of the operator $O$ at $x$. Here the quark field has a flavor index $f$ running from 1 to $N_{f}$. The expectation value $\langle\cdots\rangle$ in (40) can be taken either in the $\theta$ vacuum or in the fixed $Q$ sector, since the WT identities are valid with any external states. Combining the following two WT identities [for $O=2 m P(0)$ and $O=2 \omega(x)$ with $x \neq 0]$,

$\langle 2 m P(x) 2 m P(0)\rangle=\left\langle\partial_{\mu} A_{\mu}(x) 2 m P(0)\right\rangle+\langle 2 \omega(x) 2 m P(0)\rangle$,

$$
\langle 2 \omega(x) 2 m P(0)\rangle=\left\langle 2 \omega(x) \partial_{\mu} A_{\mu}(0)\right\rangle+\langle 2 \omega(x) 2 \omega(0)\rangle,
$$

we obtain a relation at a fixed topological charge,

$$
\begin{aligned}
\langle 2 m P(x) 2 m P(0)\rangle_{Q}= & \langle 2 \omega(x) 2 \omega(0)\rangle_{Q} \\
& +\left\langle\partial_{\mu} A_{\mu}(x) 2 m P(0)\right\rangle_{Q} \\
& +\left\langle 2 \omega(x) \partial_{\mu} A_{\mu}(0)\right\rangle_{Q} .
\end{aligned}
$$

In the large separation limit $|x| \rightarrow \infty$, using (28) the second term becomes

$$
\begin{aligned}
\left\langle\partial_{\mu} A_{\mu}(x) 2 m P(0)\right\rangle_{Q} \rightarrow & \left\langle\partial_{\mu} A_{\mu}(x)\right\rangle\langle 2 m P(0)\rangle \\
& +\frac{1}{2 V \chi_{t}}\left(1-\frac{Q^{2}}{V \chi_{t}}-\frac{c_{4}}{2 \chi_{t}^{2} V}\right) \\
& \times\left\langle\partial_{\mu} A_{\mu}(x) 2 m P(0)\right\rangle^{(2)} \\
& +\frac{1}{8 \chi_{t}^{2} V^{2}}\left\langle\partial_{\mu} A_{\mu}(x) 2 m P(0)\right\rangle^{(4)} .
\end{aligned}
$$

The first term on the right-hand side vanishes, and the second term is evaluated as

$$
\begin{aligned}
\left\langle\partial_{\mu} A_{\mu}(x) 2 m P(0)\right\rangle^{(2)} & =\left\langle\partial_{\mu} A_{\mu}(x) 2 m P(0) Q^{2}\right\rangle \\
& \rightarrow 2\left\langle\partial_{\mu} A_{\mu}(x) Q\right\rangle\langle 2 m P(0) Q\rangle .
\end{aligned}
$$

Since the translational invariance (plus an appropriate boundary condition) leads to

$$
\left\langle\partial_{\mu} A_{\mu}(x) Q\right\rangle=\frac{1}{V} \int d^{4} x\left\langle\partial_{\mu} A_{\mu}(x) Q\right\rangle=0,
$$

we obtain $\left\langle\partial_{\mu} A_{\mu}(x) 2 m P(0)\right\rangle^{(2)}=0$ in the large separation limit.
We next consider the fourth-derivative term. In general,

$$
\begin{aligned}
\langle A(x) B(0)\rangle^{(4)}= & \left\langle A(x) B(0) Q^{4}\right\rangle-6\left\langle A(x) B(0) Q^{2}\right\rangle\left\langle Q^{2}\right\rangle \\
& +\langle A(x) B(0)\rangle\left\{6\left\langle Q^{2}\right\rangle^{2}-\left\langle Q^{4}\right\rangle\right\}
\end{aligned}
$$

when $\langle A(x)\rangle=\langle B(0)\rangle=0$. Using relations

$$
\left\langle A(x) B(0) Q^{2}\right\rangle \rightarrow 2\langle A(x) Q\rangle_{c}\langle B(0) Q\rangle_{c},
$$

$$
\begin{aligned}
\left\langle A(x) B(0) Q^{4}\right\rangle= & \left\langle A(x) B(0) Q^{4}\right\rangle_{c}+\langle A(x) B(0)\rangle_{c}\left\langle Q^{4}\right\rangle_{c} \\
& +6\left\langle A(x) B(0) Q^{2}\right\rangle_{c}\left\langle Q^{2}\right\rangle_{c} \\
& +3\langle A(x) B(0)\rangle_{c}\left\langle Q^{2}\right\rangle_{c}^{2} \\
& +4\langle A(x) Q\rangle_{c}\left\langle B(0) Q^{3}\right\rangle_{c} \\
& +4\langle B(0) Q\rangle_{c}\left\langle A(x) Q^{3}\right\rangle_{c} \\
& +12\langle A(x) Q\rangle_{c}\langle B(0) Q\rangle_{c}\left\langle Q^{2}\right\rangle_{c} \\
\rightarrow & 4\langle A(x) Q\rangle_{c}\left\langle B(0) Q^{3}\right\rangle_{c} \\
& +4\langle B(0) Q\rangle_{c}\left\langle A(x) Q^{3}\right\rangle_{c} \\
& +12\langle A(x) Q\rangle_{c}\langle B(0) Q\rangle_{c}\left\langle Q^{2}\right\rangle_{c},
\end{aligned}
$$

the general formula reads

$$
\begin{aligned}
\langle A(x) B(0)\rangle^{(4)} \rightarrow & 4\langle A(x) Q\rangle_{c}\left\langle B(0) Q^{3}\right\rangle_{c}+4\langle B(0) Q\rangle_{c} \\
& \times\left\langle A(x) Q^{3}\right\rangle_{c} .
\end{aligned}
$$

For $A(x)=\partial_{\mu} A_{\mu}(x)$, the translational invariance again gives $\langle A(x) Q\rangle_{c}=\left\langle A(x) Q^{3}\right\rangle_{c}=0, \quad$ and therefore $\left\langle\partial_{\mu} A_{\mu}(x) 2 m P(0)\right\rangle^{(4)} \rightarrow 0$. We thus conclude that the term $\left\langle\partial_{\mu} A_{\mu}(x) 2 m P(0)\right\rangle_{Q}$ on the right-hand side of (43) vanishes in the large separation limit $|x| \rightarrow \infty$, and the same conclusion also holds for the last term $\left\langle 2 \omega(x) \partial_{\mu} A_{\mu}(0)\right\rangle_{Q}$ in (43).

Combining these, we finally obtain

$$
\begin{aligned}
\lim _{|x| \rightarrow \text { large }}\langle m P(x) m P(0)\rangle_{Q}= & \lim _{|x| \rightarrow \operatorname{large}}\langle\omega(x) \omega(0)\rangle_{Q} \\
= & \frac{1}{V}\left(\frac{Q^{2}}{V}-\chi_{t}-\frac{c_{4}}{2 \chi_{t} V}\right) \\
& +O\left(e^{-m_{\eta^{\prime}}|x|}\right) .
\end{aligned}
$$

Namely, the topological susceptibility can also be calculated with the singlet pseudoscalar density correlator at a fixed topology. If we apply the above evaluation to the nonsinglet WT identities, we can show

$$
\begin{aligned}
\lim _{|x| \rightarrow \text { large }}\left\langle 2 m P^{a}(x) 2 m P^{a}(0)\right\rangle_{Q} & =\lim _{|x| \rightarrow \operatorname{large}}\left\langle\partial_{\mu} A_{\mu}^{a}(x) 2 m P^{a}(0)\right\rangle_{Q} \\
& =0,
\end{aligned}
$$

where $\quad A_{\mu}^{a}(x)=\bar{\psi}(x) \gamma_{\mu} \gamma_{5} T^{a} \psi(x) \quad$ and $\quad P^{a}=$ $\bar{\psi}(x) \gamma_{5} T^{a} \psi(x)$ are the flavor nonsinglet axial-vector current and pseudoscalar density, respectively, with $\operatorname{tr} T^{a}=0$ and $\operatorname{tr}\left(T^{a} T^{b}\right)=\delta^{a b} / N_{f}$. By subtracting this equation from (51), we may write 


$$
\begin{aligned}
\lim _{|x| \rightarrow \operatorname{large}}\langle m P(x) m P(0)\rangle_{Q}^{\operatorname{disc}} \equiv & \lim _{|x| \rightarrow \operatorname{large}}\left[\langle m P(x) m P(0)\rangle_{Q}\right. \\
& \left.-\left\langle m P^{a}(x) m P^{a}(0)\right\rangle_{Q}\right] \\
= & \frac{1}{V}\left(\frac{Q^{2}}{V}-\chi_{t}-\frac{c_{4}}{2 \chi_{t} V}\right) \\
& +O\left(e^{-m_{\pi}|x|}\right),
\end{aligned}
$$

where the superscript "disc" represents a disconnected contribution, and no summation is taken here for $a$. Since the large separation limit of the nonsinglet correlator (52) is saturated only after a slowly dumping factor $e^{-m_{\pi}|x|}$ with the pion mass $m_{\pi}$, the singlet correlator (51) is preferable for practical use.

\section{B. Four-point correlation function}

Here we extend our analysis to a four-point function.

\section{Bosonic formula}

We consider a $C P$-even observable

$$
\begin{aligned}
\left\langle\omega\left(x_{1}\right) \omega\left(x_{2}\right) \omega\left(x_{3}\right) \omega\left(x_{4}\right)\right\rangle_{Q}= & \left\langle\omega\left(x_{1}\right) \omega\left(x_{2}\right) \omega\left(x_{3}\right) \omega\left(x_{4}\right)\right\rangle \\
& +\left\langle\omega\left(x_{1}\right) \omega\left(x_{2}\right) \omega\left(x_{3}\right) \omega\left(x_{4}\right)\right\rangle^{(2)} \\
& \times \frac{1}{2 V \chi_{t}}\left(1-\frac{Q^{2}}{V \chi_{t}}-\frac{c_{4}}{2 \chi_{t}^{2} V}\right) \\
& +\left\langle\omega\left(x_{1}\right) \omega\left(x_{2}\right) \omega\left(x_{3}\right) \omega\left(x_{4}\right)\right\rangle^{(4)} \\
& \times \frac{1}{8 \chi_{t}^{2} V^{2}}+O\left(V^{-3}\right) .
\end{aligned}
$$

We assume that four topological charge density operators are far apart from any others $\left(\left|x_{i}-x_{j}\right| \rightarrow\right.$ large for $\forall i \neq \forall j)$. In this limit both $\left\langle\omega\left(x_{1}\right) \omega\left(x_{2}\right) \omega\left(x_{3}\right) \omega\left(x_{4}\right)\right\rangle$ and $\left\langle\omega\left(x_{1}\right) \omega\left(x_{2}\right) \omega\left(x_{3}\right) \omega\left(x_{4}\right)\right\rangle^{(2)}$ vanish and $\left\langle\omega\left(x_{1}\right) \omega\left(x_{2}\right) \omega\left(x_{3}\right) \omega\left(x_{4}\right)\right\rangle^{(4)}$ becomes $24 \chi_{t}^{4}$, taking the procedure used to obtain the analogous formula (38) in the two-point function case. We obtain

$$
\begin{aligned}
& \left\langle\omega\left(x_{1}\right) \omega\left(x_{2}\right) \omega\left(x_{3}\right) \omega\left(x_{4}\right)\right\rangle_{Q} \\
& \quad=3 \frac{\chi_{t}^{2}}{V^{2}}\left[1+\frac{1}{\chi_{t}^{2} V}\left(c_{4}-Q^{2} \chi_{t}\right)\right]^{2}+O\left(V^{-4}\right) .
\end{aligned}
$$

In addition to the leading $O\left(1 / V^{2}\right)$ term, we also give a next-to-leading $O\left(1 / V^{3}\right)$ correction. Details of this $O\left(1 / V^{3}\right)$ calculation are given in Appendix B.

\section{Fermionic formula}

Again we use the anomalous axial WT identities, which give a chain of equations,

$$
\begin{gathered}
\left\langle P_{1} P_{2} P_{3} P_{4}\right\rangle=\left\langle d A_{1} P_{2} P_{3} P_{4}\right\rangle+\left\langle\omega_{1} P_{2} P_{3} P_{4}\right\rangle, \\
\left\langle\omega_{1} P_{2} P_{3} P_{4}\right\rangle=\left\langle d A_{2} \omega_{1} P_{3} P_{4}\right\rangle+\left\langle\omega_{1} \omega_{2} P_{3} P_{4}\right\rangle, \\
\left\langle\omega_{1} \omega_{2} P_{3} P_{4}\right\rangle=\left\langle d A_{3} \omega_{1} \omega_{2} P_{4}\right\rangle+\left\langle\omega_{1} \omega_{2} \omega_{3} P_{4}\right\rangle,
\end{gathered}
$$

$$
\left\langle\omega_{1} \omega_{2} \omega_{3} P_{4}\right\rangle=\left\langle d A_{4} \omega_{1} \omega_{2} \omega_{3}\right\rangle+\left\langle\omega_{1} \omega_{2} \omega_{3} \omega_{4}\right\rangle,
$$

where we have used a shorthand notation, $P_{i} \equiv m P\left(x_{i}\right)$, $d A_{i} \equiv \partial_{\mu} A_{\mu}\left(x_{i}\right) / 2$, and $\omega_{i} \equiv \omega\left(x_{i}\right)$. The operators are far apart from others, as before. Since the WT identities are valid in the fixed topological sector, we obtain

$$
\begin{aligned}
\left\langle P_{1} P_{2} P_{3} P_{4}\right\rangle_{Q}= & \left\langle\omega_{1} \omega_{2} \omega_{3} \omega_{4}\right\rangle_{Q}+\left\langle d A_{1} P_{2} P_{3} P_{4}\right. \\
& +\omega_{1} d A_{2} P_{3} P_{4}+\omega_{1} \omega_{2} d A_{3} P_{4} \\
& \left.+\omega_{1} \omega_{2} \omega_{3} d A_{4}\right\rangle_{Q}
\end{aligned}
$$

From the clustering property and $C P$ invariance the correlator $\left\langle d A_{i} O_{j k l}\right\rangle$ vanishes, where $O_{j k l} \equiv F_{j} F_{k} F_{l}$ with $F_{i}=$ $P_{i}$ or $\omega_{i}\left(i, j, k, l\right.$ are all different). In addition, $\left\langle d A_{i} O_{j k l}\right\rangle^{(2)}$ vanishes, because it is written as $-\left\langle d A_{i} O_{j k l} Q^{2}\right\rangle \rightarrow$ $-\left\langle d A_{i} Q\right\rangle\left\langle O_{j k l} Q\right\rangle$. Similarly, $\left\langle d A_{i} O_{j k l}\right\rangle^{(4)}$ vanishes, because it is $\left\langle d A_{i} O_{j k l} Q^{4}\right\rangle \rightarrow 4\left\langle d A_{i} Q\right\rangle\left\langle O_{j k l} Q^{3}\right\rangle$ and $\left\langle\partial_{\mu} A(x) Q\right\rangle=$ $(1 / V) \int d^{4} x\left\langle\partial_{\mu} A(x) Q\right\rangle=0$. We therefore obtain $\left\langle d A_{i} O_{j k l}\right\rangle_{Q} \rightarrow 0$, which leads to

$$
\begin{aligned}
\left\langle P_{1} P_{2} P_{3} P_{4}\right\rangle_{Q} & \rightarrow\left\langle\omega_{1} \omega_{2} \omega_{3} \omega_{4}\right\rangle_{Q} \\
& =3 \frac{\chi_{t}^{2}}{V^{2}}\left[1+\frac{1}{\chi_{t}^{2} V}\left(c_{4}-Q^{2} \chi_{t}\right)\right]^{2}+O\left(V^{-4}\right) .
\end{aligned}
$$

Similarly the nonsinglet WT identities imply that $\left\langle P_{i}^{a} P_{j}^{a} P_{k} P_{l}\right\rangle_{Q}$ and $\left\langle P_{i}^{a} P_{j}^{a} P_{k}^{b} P_{l}^{b}\right\rangle_{Q}$ vanish in the large separation limit, where $P_{i}^{a}=P^{a}\left(x_{i}\right)$ is a nonsinglet pseudoscalar density and $i, j, k, l$ are all different from others. We then conclude that the disconnected correlator has the same asymptotic form as $\left\langle\omega_{1} \omega_{2} \omega_{3} \omega_{4}\right\rangle_{Q}$ :

$$
\begin{aligned}
\left\langle P_{1} P_{2} P_{3} P_{4}\right\rangle_{Q}^{\text {disc }} \equiv & \left\langle P_{1} P_{2} P_{3} P_{4}\right\rangle_{Q}-\left\{\left\langle P_{1}^{a} P_{2}^{a} P_{3} P_{4}\right\rangle_{Q}\right. \\
& \left.+\left\langle P_{1} P_{2} P_{3}^{a} P_{4}^{a}\right\rangle_{Q}-\left\langle P_{1}^{a} P_{2}^{a} P_{3}^{b} P_{4}^{b}\right\rangle_{Q}\right\} \\
& -\{2 \leftrightarrow 3\}-\{2 \leftrightarrow 4\} \\
\rightarrow & 3 \frac{\chi_{t}^{2}}{V^{2}}\left[1+\frac{1}{\chi_{t}^{2} V}\left(c_{4}-Q^{2} \chi_{t}\right)\right]^{2} \\
& +O\left(V^{-4}\right),
\end{aligned}
$$

where no sum is taken for $a \neq b$.

The formulas (55) and (62) provide another method to extract the topological susceptibility from a fixed topological sector. Since the contamination from the higher order effect in $1 / V$ with the unknown constant $c_{4}$ appears with a different coefficient from the two-point case (39) and (51), both $\chi_{t}$ and $c_{4}$ can be extracted by combining the two- and four-point correlators. Note that this calculation is free from short-distance singularities, because the operators are explicitly put apart from others. This is in contrast to the usual definition $\chi_{t}=(1 / V) \int d^{4} x\langle\omega(x) \omega(0)\rangle$ (at a fixed $\theta$ ). 


\section{Three-point correlation function}

Topological susceptibility can be extracted also from $C P$-odd observables. The simplest case is given by a three-point function as

$$
\begin{aligned}
& \lim _{\left|x_{i}-x_{j}\right| \rightarrow \operatorname{large}}\left\langle\omega\left(x_{1}\right) \omega\left(x_{2}\right) \omega\left(x_{3}\right)\right\rangle_{Q} \\
= & \lim _{\left|x_{i}-x_{j}\right| \rightarrow \operatorname{large}}\left\langle\omega\left(x_{1}\right) \omega\left(x_{2}\right) \omega\left(x_{3}\right)\right\rangle^{(3)} \frac{i Q}{2 \chi_{t}^{2} V^{2}}+O\left(V^{-3}\right) \\
= & -\lim _{\left|x_{i}-x_{j}\right| \rightarrow \operatorname{large}}\left\langle\omega\left(x_{1}\right) \omega\left(x_{2}\right) \omega\left(x_{3}\right) Q^{3}\right\rangle \frac{Q}{2 \chi_{t}^{2} V^{2}}+O\left(V^{-3}\right) \\
= & -3 \chi_{t} \frac{Q}{V^{2}}\left[1+\frac{7 c_{4}}{6 \chi_{t}^{2} V}-\frac{Q^{2}}{3 \chi_{t} V}\right]+O\left(V^{-4}\right) .
\end{aligned}
$$

As in the cases of two- and four-point functions we may rewrite the above result in terms of the fermionic quantities,

$$
\begin{aligned}
& \lim _{\left|x_{i}-x_{j}\right| \rightarrow \operatorname{large}}\left\langle m P\left(x_{1}\right) m P\left(x_{2}\right) m P\left(x_{3}\right)\right\rangle_{Q} \\
= & \lim _{\left|x_{i}-x_{j}\right| \rightarrow \operatorname{large}}\left\langle m P\left(x_{1}\right) m P\left(x_{2}\right) m P\left(x_{3}\right)\right)_{Q}^{d_{i s c}} \\
= & \lim _{\left|x_{i}-x_{j}\right| \rightarrow \operatorname{large}}\left\langle\omega\left(x_{1}\right) \omega\left(x_{2}\right) \omega\left(x_{3}\right)\right\rangle_{Q} \\
= & -3 \chi_{t} \frac{Q}{V^{2}}\left[1+\frac{7 c_{4}}{6 \chi_{t}^{2} V}-\frac{Q^{2}}{3 \chi_{t} V}\right]+O\left(V^{-4}\right) .
\end{aligned}
$$

As expected, the three-point function is useful only when the net topological charge is nonzero.

\section{CP-ODD ELECTROMAGNETIC FORM FACTOR}

The three-point function considered above is an example of a $C P$-odd observable. In our formulation, as the formula (29) implies, the $C P$-odd observable (or its first derivative with respect to $\theta$ ) can be calculated in a (nonzero) fixed topological sector. Another interesting example of this class is the neutron electric dipole moment.

The $C P$-odd electromagnetic form factor $F_{3}\left(q^{2}\right)$ is related to the neutron electric dipole moment $d_{N}$ as

$$
d_{N}=\lim _{q^{2} \rightarrow 0} \frac{F_{3}\left(q^{2}\right)}{2 m_{N}},
$$

where $q$ is a momentum transfer. It has been shown in [21] that $F_{3}\left(q^{2}\right)$ can be extracted from the correlation functions $\left\langle\bar{N}(p) J_{\mu}^{\mathrm{EM}}(q) N\left(p^{\prime}\right) Q\right\rangle$ and $\langle\bar{N}(p) N(p) Q\rangle$, where $N(p)$ is a neutron interpolating field and $J_{\mu}^{\mathrm{EM}}$ is the electromagnetic current. The momentum transfer is denoted by $q=p-$ $p^{\prime}$.

Once $\chi_{t}$ is extracted from the methods explained in the previous section, these neutron correlators can be extracted at a fixed topological charge using (29),

$$
\begin{aligned}
\left\langle\bar{N}(p) J_{\mu}^{\mathrm{EM}}(q) N\left(p^{\prime}\right)\right\rangle_{Q}^{\mathrm{odd}}= & \left\langle\bar{N}(p) J_{\mu}^{\mathrm{EM}}(q) N\left(p^{\prime}\right) Q\right\rangle \frac{Q}{\chi_{t} V} \\
& +O\left(V^{-2}\right)
\end{aligned}
$$

$$
\langle\bar{N}(p) N(p)\rangle_{Q}^{\text {odd }}=\langle\bar{N}(p) N(p) Q\rangle \frac{Q}{\chi_{t} V}+O\left(V^{-2}\right),
$$

where the superscript "odd" means that a $C P$-odd part of the correlation function is considered.

\section{CONCLUSIONS AND DISCUSSIONS}

In this paper, we have derived general formulas which express arbitrary correlation functions at a fixed topological charge $Q$ in terms of the same correlation function (and its derivatives) in the $\theta$ vacuum. As expected from the intuitive argument of local topological excitations, the difference between the fixed $Q$ vacuum and the fixed $\theta$ vacuum disappears in the large volume limit as $1 / V$. The relation is established only using fundamental properties of the quantum field theory, such as the cluster decomposition principle.

These formulas open a new possibility to calculate physical quantities in the lattice QCD simulations at a fixed topological charge. This will become unavoidable as the continuum limit is approached, irrespective of the lattice fermion formulation one employs, as far as the algorithm is based on the continuous evolution of the gauge field. (A proposal to avoid this limitation has recently been proposed [22]. Its numerical feasibility is yet to be investigated.)

For the correlators of the local topological charge operator, the $1 / V$ expansion is worked out for two-, three-, and four-point functions to the second nontrivial order. The local topological charge operator may either be a bosonic one or a fermionic singlet pseudoscalar operator. At the leading nontrivial order the topological susceptibility $\chi_{t}$ appears as an expansion parameter, and a higher order parameter $c_{4}$ appears at the second order. In principle, these parameters can be determined by the lattice data. The different correlators and different $V$ and $Q$ may be used to check the results. This method is free from shortdistance singularities, since the local topological charge operators are put apart from others and no contact term appears. Numerical calculation is in progress by the JLQCD Collaboration on the gauge configurations generated with a dynamical overlap fermion [5-8]. Once these parameters are numerically obtained, they can be used as input parameters for other physical observables.

The limitation of the formulas comes from the use of the saddle point expansion. It requires that the volume is large enough, $\chi_{t} V \gg 1$, that the local topological fluctuation is, in fact, active. It corresponds to the condition $\left\langle 0\left|Q^{2}\right| 0\right\rangle \gg$ 1 for that volume. In addition, in order that the saddle point $\theta_{c} \simeq Q /\left(\chi_{t} V\right)$ can be expanded around $\theta=0$, the (fixed) topological charge $|Q|$ must be much smaller than 
$\chi_{t} V=\left\langle 0\left|Q^{2}\right| 0\right\rangle$. Since $\chi_{t}$ is estimated in ChPT as $\chi_{t}=$ $m \Sigma / N_{f}$ for $N_{f}$ flavors of sea quarks, the condition is $|Q| \ll m \Sigma V$. This means that the system must be well separated from the $\epsilon$ regime $(m \Sigma V \lesssim 1)$. This makes sense, because in the $\epsilon$ regime the physical quantities have substantial dependence on the topological charge and cannot be simply expressed by the saddle point expansion.

Our general formulas can also be applied to other observables, such as masses, decay constants, and matrix elements. Although the knowledge of the $\theta$ dependence of the observables is needed, only a few derivatives with respect to $\theta$ are sufficient for small $\theta$, which is physically most relevant. Using the systematic $1 / V$ expansion, one can, in principle, extract those derivatives to arbitrary finite order by looking at the $Q$ dependence. Combining them with $\chi_{t}, c_{4}, \ldots$, the physical observables in the small $\theta$ vacuum can be reconstructed. This is not surprising, because if we were able to determine all the coefficients in $E(\theta)$, we could compute observables in the $\theta$ vacuum by a reweighting method. The point of our work is to present a practically feasible strategy in which the computational effort is drastically reduced. For the quantities well described by ChPT, estimation of the $\theta$ dependence based on the chiral Lagrangian is possible. Some results at the leading order were obtained in [12], and we are extending them to the next-to-leading order. This can also be used as an independent consistency check.

Once $\chi_{t}$ is extracted, we can calculate the first derivative of the $C P$-odd quantities from simulations at a fixed nonzero topological charge using (29). The most interesting such quantity in the context of lattice QCD calculation is the neutron electric dipole moment. For this quantity, the calculation of the $C P$-odd form factor in the fixed nonzero topological charge suffices to predict the physical result in the $\theta$ vacuum.

\section{ACKNOWLEDGMENTS}

The authors would like to acknowledge the workshop at Yukawa Institute YITP-W-05-25 "Actions and Symmetries in Lattice Gauge Theories," where part of this work was initiated. S. A. would like thank Professor K.F. Liu for useful discussions. This work is supported in part by the Grants-in-Aid for Scientific Research from the Ministry of Education, Culture, Sports, Science and Technology (No. 13135204, No. 15204015, No. 15540251, No. 16028201, No. 18034011, No. 18340075, No. 18840045 , No. 19540286).

\section{APPENDIX A: COMPARISON OF THE SADDLE POINT EXPANSION WITH AN EXACT $\theta$ INTEGRATION}

In this appendix we demonstrate that the saddle point expansion reproduces the results from the exact $\theta$ integration, by considering a simple model for $E(\theta)$.

We consider the following form of the $\theta$ dependence:

$$
E(\theta)=\chi_{t}[1-\cos \theta] .
$$

The partition function $Z_{Q}$ can be exactly calculated as

$$
Z_{Q}=e^{-V \chi_{t}} I_{Q}\left(V \chi_{t}\right),
$$

where $I_{Q}$ is the modified Bessel function. If the form of $G(\theta)$ is given, $G_{Q}$ can also be calculated exactly. For example, let us consider the case that $G(\theta)=\langle\theta|Q| \theta\rangle$ or $\left\langle\theta\left|Q^{2}\right| \theta\right\rangle$. From $\langle\theta|Q| \theta\rangle=-i V d E / d \theta=-i V \chi_{t} \sin \theta$, we have

$$
\begin{aligned}
\langle Q\rangle_{Q} & =\frac{-i}{Z_{Q}} \int_{-\pi}^{\pi} \frac{d \theta}{2 \pi} V \chi_{t} \sin \theta e^{-V E(\theta)+i \theta Q} \\
& =V \chi_{t} \frac{I_{Q-1}\left(V \chi_{t}\right)-I_{Q+1}\left(V \chi_{t}\right)}{2 I_{Q}\left(V \chi_{t}\right)} .
\end{aligned}
$$

Using a formula for the Bessel function $I_{n-1}(z)-$ $I_{n+1}(z)=(2 n / z) I_{n}(z)$, the correct result $\langle Q\rangle_{Q}=Q$ is reproduced. Similarly, using

$$
\begin{aligned}
\left\langle\theta\left|Q^{2}\right| \theta\right\rangle & =\frac{d^{2}}{d \theta^{2}} \log Z(\theta)+\langle\theta|Q| \theta\rangle^{2} \\
& =V \chi_{t} \cos \theta-\left(V \chi_{t} \sin \theta\right)^{2},
\end{aligned}
$$

we obtain an expression

$$
\left\langle Q^{2}\right\rangle_{Q}=\frac{z\left[I_{Q+1}\left(V \chi_{t}\right)+I_{Q-1}\left(V \chi_{t}\right)\right]+\left(z^{2} / 2\right)\left[I_{Q+2}\left(V \chi_{t}\right)+I_{Q-2}\left(V \chi_{t}\right)-2 I_{Q}\left(V \chi_{t}\right)\right]}{2 I_{Q}\left(V \chi_{t}\right)},
$$

which leads to the correct result $\left\langle Q^{2}\right\rangle_{Q}=Q^{2}$.

We now consider a general $G(\theta)$. We will calculate $G_{Q}$, using the expansion of $G(\theta)$,

$$
G_{Q}=G(0)+\sum_{n=1}^{\infty} \frac{G^{(n)}}{n !}\left\langle\theta^{n}\right\rangle,
$$

where

$$
\left\langle\theta^{n}\right\rangle_{Q}=\frac{1}{Z_{Q}} \int_{-\pi}^{\pi} \frac{d \theta}{2 \pi} Z(\theta) e^{i \theta Q} \theta^{n} .
$$

This expansion can be evaluated using the connected part

$$
\left\langle(i \theta)^{n}\right\rangle_{Q, c}=\frac{d^{n}}{d Q^{n}} \log Z_{Q}
$$

and the formulas 


$$
\begin{gathered}
\langle X\rangle=\langle X\rangle_{c}, \\
\left\langle X^{2}\right\rangle=\left\langle X^{2}\right\rangle_{c}+\langle X\rangle^{2}, \\
\left\langle X^{3}\right\rangle=\left\langle X^{3}\right\rangle_{c}+3\left\langle X^{2}\right\rangle_{c}\langle X\rangle+\langle X\rangle^{3}, \\
\left\langle X^{4}\right\rangle=\left\langle X^{4}\right\rangle_{c}+4\left\langle X^{3}\right\rangle_{c}\langle X\rangle+3\left(\left\langle X^{2}\right\rangle_{c}\right)^{2} \\
+6\left\langle X^{2}\right\rangle_{c}\langle X\rangle^{2}+\langle X\rangle^{4},
\end{gathered}
$$

with $X=\theta$ in our case. We evaluate the $Q$ derivatives in the large $V$ limit, using the asymptotic expansion of the Bessel function

$$
I_{Q}(z) \simeq e^{z} \frac{1}{\sqrt{2 \pi Z}} \sum_{n=0}^{\infty}(-1)^{n}(Q, n) \frac{1}{(2 z)^{n}}+O\left(e^{-z}\right),
$$

where $z=\chi_{t} V$ and

$$
\begin{aligned}
& (Q, n)=\frac{\left(4 Q^{2}-1^{2}\right)\left(4 Q^{2}-3^{2}\right) \cdots\left(4 Q^{2}-(2 n-1)^{2}\right)}{n ! 2^{2 n}}, \\
& (Q, 0)=1 .
\end{aligned}
$$

Using these, the partition function is written as

$$
Z_{Q} \simeq \frac{1}{\sqrt{2 \pi z}}\left[1-\frac{(Q, 1)}{2 z}+\frac{(Q, 2)}{(2 z)^{2}}+\cdots\right]
$$

and we obtain, ignoring $O\left(V^{-3}\right)$ contributions,

$$
\begin{aligned}
\log Z_{Q} & =-\log \sqrt{2 \pi z}-\frac{1}{2 z}\left[(Q, 1)+\frac{(Q, 1)^{2}}{2(2 z)}-\frac{(Q, 2)}{2 z}\right] \\
& =-\log \sqrt{2 \pi z}-\frac{1}{2 z}\left(Q^{2}-\frac{1}{4}\right)\left(1+\frac{1}{2 z}\right),
\end{aligned}
$$

from which

$$
\begin{gathered}
\frac{d}{d Q} \log Z_{Q}=-\frac{Q}{z}\left(1+\frac{1}{2 z}\right), \\
\frac{d^{2}}{d Q^{2}} \log Z_{Q}=-\frac{1}{z}\left(1+\frac{1}{2 z}\right), \\
\frac{d^{n}}{d Q^{n}} \log Z_{Q}=0, \quad n=3,4,5, \cdots .
\end{gathered}
$$

Therefore,

$$
\begin{gathered}
\langle\theta\rangle_{Q}=i \frac{Q}{z}\left(1+\frac{1}{2 z}\right), \\
\left\langle\theta^{2}\right\rangle_{Q}=\frac{1}{z}\left(1+\frac{1}{2 z}\right)-\frac{Q^{2}}{z^{2}}+O\left(z^{-3}\right), \\
\left\langle\theta^{3}\right\rangle_{Q}=i \frac{3 Q}{z^{2}}+O\left(z^{-3}\right), \\
\left\langle\theta^{4}\right\rangle_{Q}=\frac{3}{z^{2}}+O\left(z^{-3}\right) .
\end{gathered}
$$

Collecting these, the final result becomes

$$
\begin{aligned}
G_{Q}= & G(0)+G^{(2)}(0) \frac{1}{2 z}\left(1+\frac{1}{2 z}-\frac{Q^{2}}{z}\right)+G^{(4)}(0) \frac{1}{8 z^{2}} \\
& +G^{(1)}(0) i \frac{Q}{z}\left(1+\frac{1}{2 z}\right)+G^{(3)}(0) i \frac{Q}{2 z^{2}} .
\end{aligned}
$$

Noticing that $c_{4}=-\chi_{t}$ for the present case, this result completely agrees with the previous result (27) within the $O\left(V^{-3}\right)$ errors. This demonstrates that the saddle point expansion reproduces correct results, up to exponentially small corrections.

The leading order of the chiral perturbation theory gives

$$
E(\theta)=N_{f}^{2} \chi_{t}\left[1-\cos \left(\theta / N_{f}\right)\right]
$$

In this case, we can extend the integration range to $-N_{f} \pi<\theta<N_{f} \pi$ by ignoring exponentially small contributions. After performing $\theta$ integration exactly, we obtain

$$
Z_{Q}=e^{-z} I_{\hat{Q}}(z)+O\left(e^{-z\left[1-\cos \left(\pi / N_{f}\right)\right]}\right)
$$

where $z=V N_{f}^{2} \chi_{t}$ and $\hat{Q}=N_{f}^{2} Q$. Noticing that $c_{4}=$ $-\chi_{t} / N_{f}^{2}$ in this case, it is easy to see that the saddle point expansion agrees with exact results order by order in the $1 / V$ expansion, as long as exponentially small corrections are ignored.

\section{APPENDIX B: CONTRIBUTIONS AT $V^{-3}$}

\section{1. $C P$-even contributions}

For $\theta_{c}=O\left(V^{-1}\right), C P$-even contributions become

$$
\begin{aligned}
G_{Q}^{\text {even }}= & G+G^{(2)} \frac{\theta_{c}^{2}}{2}+G^{(2)} \frac{1}{2 x}\left(1-\frac{E^{(4)} V}{2 x^{2}}-\frac{E^{(6)} V}{8 x^{3}}\right. \\
& \left.+\frac{2\left(E^{(4)} V\right)^{2}}{3 x^{4}}+\frac{5\left(E^{(3)} V\right)^{2}}{4 x^{3}}\right)+G^{(4)} \frac{\theta_{c}^{2}}{4 x} \\
& +G^{(4)} \frac{1}{4 !}\left(\frac{3}{x^{2}}-\frac{4 E^{(4)} V}{x^{4}}\right)+G^{(6)} \frac{15}{6 ! x^{3}} \\
& -G^{(2)} \theta_{c} \frac{E^{(3)} V}{2 x^{2}}+O\left(V^{-4}\right) .
\end{aligned}
$$

Using the large separation limit $G=G^{(2)}=0$, $G^{(4)}=24 \chi_{t}^{4}, \quad$ and $\quad G^{(6)}=480 \chi_{t}^{3} c_{4} \quad$ for $\quad G=$ $\left\langle\omega\left(x_{1}\right) \omega\left(x_{2}\right) \omega\left(x_{3}\right) \omega\left(x_{4}\right)\right\rangle$, we obtain

$$
\begin{aligned}
\left\langle\omega\left(x_{1}\right) \omega\left(x_{2}\right) \omega\left(x_{3}\right) \omega\left(x_{4}\right)\right\rangle_{Q} \rightarrow & \frac{3 \chi_{t}^{2}}{V^{2}}\left[1+\frac{2}{\chi_{t}^{2} V}\left(c_{4}-Q^{2} \chi_{t}\right)\right] \\
& +O\left(V^{-4}\right) .
\end{aligned}
$$




\section{2. $C P$-odd contributions}

For $\theta_{c}=O\left(V^{-1}\right), C P$-odd contributions become

$$
\begin{aligned}
G_{Q}^{\text {odd }}= & G^{(1)} \theta_{c}+G^{(3)} \frac{\theta_{c}^{3}}{3 !}+G^{(3)} \frac{\theta_{c}}{2 x}\left(1-\frac{E^{(4)} V}{2 x^{2}}\right) \\
& +G^{(5)} \frac{3 \theta_{c}}{4 ! x^{2}}-G^{(1)}\left[\frac{E^{(3)} V}{2 x^{2}}\left(1-\frac{4 E^{(4)} V}{3 x^{2}}\right)+\frac{E^{5} V}{8 x^{3}}\right] \\
& -G^{(3)} \frac{5 E^{(3)} V}{12 x^{3}} .
\end{aligned}
$$

Using the large separation limit $G^{(1)}=0, G^{(3)}=i 6 \chi_{t}^{3}$, and $G^{(5)}=i 60 \chi_{t}^{2} c_{4}$ for $G=\left\langle\omega\left(x_{1}\right) \omega\left(x_{2}\right) \omega\left(x_{3}\right)\right\rangle$, we obtain
[1] S. Weinberg, The Quantum Theory of Fields (Cambridge University Press, Cambridge, England, 1996), Vol. II, p. 455.

[2] S. Duane, A. D. Kennedy, B. J. Pendleton, and D. Roweth, Phys. Lett. B 195, 216 (1987).

[3] B. Alles, G. Boyd, M. D'Elia, A. Di Giacomo, and E. Vicari, Phys. Lett. B 389, 107 (1996).

[4] L. Del Debbio, G. M. Manca, and E. Vicari, Phys. Lett. B 594, 315 (2004).

[5] T. Kaneko et al. (JLQCD Collaboration), Proc. Sci., LAT2006 (2006) 054 [arXiv:hep-lat/0610036].

[6] S. Hashimoto et al. (JLQCD Collaboration), Proc. Sci., LAT2006 (2006) 052 [arXiv:hep-lat/0610011].

[7] H. Matsufuru et al. (JLQCD Collaboration), Proc. Sci., LAT2006 (2006) 031 [arXiv:hep-lat/0610026].

[8] N. Yamada et al. (JLQCD Collaboration), Proc. Sci. LAT2006 (2006) 060 [arXiv:hep-lat/0609073].

[9] H. Fukaya, S. Hashimoto, K. I. Ishikawa, T. Kaneko, H. Matsufuru, T. Onogi, and N. Yamada (JLQCD Collaboration), Phys. Rev. D 74, 094505 (2006).
[10] H. Fukaya et al. (JLQCD Collaboration), Phys. Rev. Lett. 98, 172001 (2007).

[11] H. Fukaya et al., arXiv:0705.3322 [Phys. Rev. D (to be published)].

[12] R. Brower, S. Chandrasekharan, J. W. Negele, and U. J. Wiese, Phys. Lett. B 560, 64 (2003).

[13] C. Vafa and E. Witten, Phys. Rev. Lett. 53, 535 (1984).

[14] E. Witten, Ann. Phys. (N.Y.) 128, 363 (1980).

[15] L. Del Debbio, H. Panagopoulos, and E. Vicari, J. High Energy Phys. 08 (2002) 044.

[16] M. D'Elia, Nucl. Phys. B661, 139 (2003).

[17] L. Giusti, S. Petrarca, and B. Taglienti, arXiv:0705.2352.

[18] L. Del Debbio, H. Panagopoulos, and E. Vicari, arXiv:0706.1479.

[19] H. Leutwyler and A. Smilga, Phys. Rev. D 46, 5607 (1992).

[20] H. Fukaya and T. Onogi, Phys. Rev. D 70, 054508 (2004).

[21] E. Shintani et al., Phys. Rev. D 72, 014504 (2005).

[22] M. Golterman and Y. Shamir, arXiv:0705.2928. 